\title{
Existence results for third-order impulsive neutral differential equations with deviating arguments
}

\author{
Guobing Ye*, Yulin Zhao and Li Huang
}

"Correspondence:

yeguobing19@sina.com College of Science, Hunan

University of Technology, Zhuzhou,

Hunan 412007, P.R. China

\begin{abstract}
By using the fixed-point theorem of Leray-Schauder or Banach, we discuss the existence of solutions for third-order impulsive neutral differential equations with deviating arguments. Two examples are given to demonstrate our main results. MSC: $34 \mathrm{~A} 37 ; 34 \mathrm{k} 05$
\end{abstract}

Keywords: existence; impulsive neutral differential equation; third-order; fixed point

\section{Introduction}

Impulsive differential equations are a class of important models which describe many evolution processes that abruptly change their state at a certain moment. The theory of impulsive differential equations has become an active area of investigation in recent years due to their numerous applications for problems arising in mechanics, electrical engineering, medicine biology, ecology, and other areas of science (see, for example, [1-3] and the references therein). With regard to ordinary impulsive differential equations, we have refer to some work $[1,4-6]$. Partial neutral differential equations with infinite delay have been used for modeling the evolution of physical systems in which the response of the system depends on the current state as well as the past history of the system (see, for instance, [7, $8]$ on the description of heat conduction in materials with fading memory). First-order abstract neutral functional differential equations with finite delay have been studied in [9-11] among others. The work on first-order abstract neutral functional differential equations with unbounded delay was initiated in $[12,13]$. Relative to second-order abstract neutral differential equations, we must mention [14]. Recently, weighted pseudo almost periodic solutions to some partial neutral functional differential equations have been considered in [15]; Baghli et al. [16] have investigated sufficient conditions for the existence of mild solutions, on the positive half-line, for two classes of first-order functional and neutral functional perturbed differential equations with infinite delay. Hernández et al. [17, 18] have studied the existence of mild solutions for a class of autonomous impulsive partial neutral functional differential equations with infinite delay of first and second order. The authors in [19-30] have further investigated the existence results for many kinds of impulsive neutral differential equations.

@2014 Ye et al.; licensee Springer. This is an Open Access article distributed under the terms of the Creative Commons Attribution License (http://creativecommons.org/licenses/by/2.0), which permits unrestricted use, distribution, and reproduction in any medium, provided the original work is properly cited. 
In [19-30], the neutral terms of those equations are

$$
\begin{aligned}
& \frac{d}{d t}\left[x(t)-g\left(t, x_{t}\right)\right], \\
& \frac{d}{d t}\left[x(t)-F\left(t, x(t), x\left(b_{1}(t)\right), \ldots, x\left(b_{m}(t)\right)\right)\right], \\
& \frac{d}{d t}\left[x(t)-g\left(t, x_{t}, \int_{0}^{t} a\left(t, s, x_{s}\right) d s\right)\right] \text { and } \\
& \frac{d^{2}}{d t^{2}}\left[x(t)-g\left(t, x_{t}\right)\right],
\end{aligned}
$$

respectively.

The histories $x_{t}:(-\infty, 0] \rightarrow X, x_{t}(s)=x(t+s), s \leq 0$, belong to an abstract phase space $\mathcal{B}_{h}$.

Motivated by [19-30], we will consider the case that the neutral term of the equation is $u^{\prime \prime \prime}(\theta(t))$. Obviously, the neutral term is different from the previous literature. Besides, the authors in $[17,19-30]$ have studied the impulsive neutral differential equations of the first or the second order, but, in this paper, we focus on the third-order impulsive neutral differential equations. Here, we introduce a real Banach space, which has been adopted by us the first time.

Our results are based on the fixed-point theorem of Leray-Schauder or Banach.

Consider the following impulsive neutral differential equations:

$$
\left\{\begin{array}{l}
u^{\prime \prime \prime}(\theta(t))=f\left(t, u(t), u^{\prime}(t), u^{\prime \prime}(t), u\left(\varphi_{1}(t)\right), u\left(\varphi_{2}(t)\right)\right) \\
\quad t \in J=[0, a], t \neq \theta^{-1}\left(t_{k}\right), \\
\Delta u^{(i)}\left(t_{k}\right)=I_{i k}\left(u^{(i)}\left(t_{k}\right)\right), \quad i=0,1,2, k=1, \ldots, p \\
u^{(i)}(0)=u_{0}^{(i)}, \quad i=0,1,2
\end{array}\right.
$$

where $0=t_{0}<t_{1}<\cdots<t_{p}<t_{p+1}=a, I_{i k} \in C(\mathbf{R}, \mathbf{R}), \Delta u^{(i)}\left(t_{k}\right)=u^{(i)}\left(t_{k}^{+}\right)-u^{(i)}\left(t_{k}\right)(i=0,1,2)$. Throughout this paper, we assume $\theta \in C^{3}(J, \mathbf{R}), \theta$ is monotone increasing with respect to $t, 0 \leq \theta(t) \leq t(t \in J), \theta(0)=0$, and $\theta(a)=a$. Let $\theta^{\prime}(t)>0$ with $\theta^{-1} \in C^{3}(J, \mathbf{R}), \bar{J}=$ $J \backslash\left\{\theta^{-1}\left(t_{1}\right), \ldots, \theta^{-1}\left(t_{p}\right)\right\}$. Also let $f: J \times \mathbf{R}^{5} \rightarrow \mathbf{R}$ be continuous everywhere except for some $\left\{\theta^{-1}\left(t_{k}\right)\right\} \times \mathbf{R}^{5}$ at which $f\left(\theta^{-1}\left(t_{k}\right)^{+}, x_{0}, x_{1}, x_{2}, y_{1}, y_{2}\right)$ and $f\left(\theta^{-1}\left(t_{k}\right)^{-}, x_{0}, x_{1}, x_{2}, y_{1}, y_{2}\right)$ exist and $f\left(\theta^{-1}\left(t_{k}\right)^{-}, x_{0}, x_{1}, x_{2}, y_{1}, y_{2}\right)=f\left(\theta^{-1}\left(t_{k}\right), x_{0}, x_{1}, x_{2}, y_{1}, y_{2}\right) . \varphi_{i} \in C(J, \mathbf{R})$ with $\varphi_{i}(J) \subseteq J(i=1,2)$. Denote by $P C(X, Y)$, where $X \subset \mathbf{R}, Y \subset \mathbf{R}$, the set of all functions $u: X \rightarrow Y$ which are piecewise continuous in $X$ with points of discontinuity of the first kind at the points $t_{k} \in X$, where the limits $u\left(t_{k}^{+}\right)<\infty$ and $u\left(t_{k}^{-}\right)=u\left(t_{k}\right)<\infty$.

\section{Preliminaries}

Note that $m_{11}=\min _{t \in J} \theta^{\prime}(t), \quad m_{12}=\max _{t \in J} \theta^{\prime}(t), \quad m_{21}=\min _{t \in J}\left|\theta^{\prime \prime}(t)\right|$, and $m_{22}=$ $\max _{t \in J}\left|\theta^{\prime \prime}(t)\right|$. It is clear that $m_{11}>0$ and $m_{21} \geq 0$.

Let $E_{0}=\left\{u \mid u, u^{\prime}, u^{\prime \prime} \in P C(J, \mathbf{R})\right\}$. Evidently, $E_{0}$ is a real Banach space with norm

$$
\|u(t)\|_{E_{0}}=\max \left\{\|u(t)\|_{P C},\left\|u^{\prime}(t)\right\|_{P C},\left\|u^{\prime \prime}(t)\right\|_{P C}\right\},
$$

where

$$
\|u(t)\|_{P C}=\sup _{t \in J}|u(t)|, \quad\left\|u^{\prime}(t)\right\|_{P C}=\sup _{t \in J}\left|u^{\prime}(t)\right|, \quad\left\|u^{\prime \prime}(t)\right\|_{P C}=\sup _{t \in J}\left|u^{\prime \prime}(t)\right| .
$$


Further, let $E=\left\{u(\theta(t)) \mid u(t) \in E_{0}\right\}$. We can check that $E$ is also a real Banach space with norm

$$
\|u(\theta(t))\|=\max \left\{\|u(\theta(t))\|_{P C^{\prime}},\left\|u^{\prime}(\theta(t))\right\|_{P C^{*}},\left\|u^{\prime \prime}(\theta(t))\right\|_{P C^{* *}}\right\}
$$

where

$$
\begin{aligned}
& \|u(\theta(t))\|_{P C}=\sup _{t \in J}|u(\theta(t))|=\sup _{t \in J}|u(t)|=\|u(t)\|_{P C^{\prime}} \\
& \left\|u^{\prime}(\theta(t))\right\|_{P C^{*}}=\sup _{\theta(t) \in J}\left|\frac{d u(\theta(t))}{d \theta(t)}\right| \cdot \sup _{t \in J} \frac{d \theta}{d t}=\sup _{t \in J}\left|u^{\prime}(t)\right| \cdot m_{12}=m_{12}\left\|u^{\prime}(t)\right\|_{P C^{\prime}} \\
& \left\|u^{\prime \prime}(\theta(t))\right\|_{P C^{* * *}}=\sup _{\theta(t) \in J}\left|\frac{d^{2} u(\theta(t))}{\theta^{2}(t)}\right| \cdot \sup _{t \in J}\left(\frac{d \theta}{d t}\right)^{2}=\sup _{t \in J}\left|u^{\prime \prime}(t)\right| \cdot m_{12}^{2}=m_{12}^{2}\left\|u^{\prime \prime}(t)\right\|_{P C^{\prime}} .
\end{aligned}
$$

Define the operator $B: u(t) \longmapsto u(\theta(t))$, where $u(t) \in E_{0}$ and $u(\theta(t)) \in E$. It is evident that $B$ is topological linear isomorphic, which implies that $E$ is a real Banach space.

Since $\frac{\theta(a)-\theta(0)}{a-0}=\theta^{\prime}(\bar{t})(0<\bar{t}<a)$, i.e., $\theta^{\prime}(\bar{t})=1$, we get $m_{12} \geq 1$, next we have

$$
\left\|u^{\prime}(\theta(t))\right\|_{P C^{*}}=m_{12}\left\|u^{\prime}(t)\right\|_{P C} \geq\left\|u^{\prime}(t)\right\|_{P C^{*}}
$$

Similarly, we have

$$
\left\|u^{\prime \prime}(\theta(t))\right\|_{P C^{* *}} \geq\left\|u^{\prime \prime}(t)\right\|_{P C}
$$

So

$$
\|u(t)\|_{E_{0}} \leq\|u(\theta(t))\| .
$$

Lemma 1 Let $u \in E_{0}$.

Then $u$ is a solution of (1.1) if and only if $u(t) \in E_{0}$ is a solution of the following integral equation:

$$
\begin{aligned}
u(\theta(t))= & \sum_{i=0}^{2} \frac{u_{0}^{(i)}}{i !} t^{i}+\frac{1}{2} \int_{0}^{t}(t-s)^{2} f\left(s, u(s), u^{\prime}(s), u^{\prime \prime}(s), u\left(\varphi_{1}(s)\right), u\left(\varphi_{2}(s)\right)\right) d s \\
& +\sum_{0<\theta^{-1}\left(t_{k}\right)<t} \sum_{i=0}^{2} \frac{\left(t-\theta^{-1}\left(t_{k}\right)\right)^{i}}{i !} I_{i k}\left(u^{(i)}\left(t_{k}\right)\right), \quad t \in J .
\end{aligned}
$$

Proof (i) Necessity.

For $\theta^{-1}\left(t_{k}\right)<t \leq \theta^{-1}\left(t_{k+1}\right)(k=0,1, \ldots, p)$, by (1.1), we get

$$
\begin{aligned}
& u\left(t_{1}\right)-u(0)=u\left(\theta\left(\theta^{-1}\left(t_{1}\right)\right)\right)-u(\theta(0))=\int_{0}^{\theta^{-1}\left(t_{1}\right)} u^{\prime}(\theta(s)) d s, \\
& u\left(t_{2}\right)-u\left(t_{1}^{+}\right)=u\left(\theta\left(\theta^{-1}\left(t_{2}\right)\right)\right)-u\left(\theta\left(\theta^{-1}\left(t_{1}\right)^{+}\right)\right)=\int_{\theta^{-1}\left(t_{1}\right)}^{\theta^{-1}\left(t_{2}\right)} u^{\prime}(\theta(s)) d s,
\end{aligned}
$$




$$
\begin{aligned}
& u\left(t_{k}\right)-u\left(t_{k-1}^{+}\right)=u\left(\theta\left(\theta^{-1}\left(t_{k}\right)\right)\right)-u\left(\theta\left(\theta^{-1}\left(t_{k-1}\right)^{+}\right)\right)=\int_{\theta^{-1}\left(t_{k-1}\right)}^{\theta^{-1}\left(t_{k}\right)} u^{\prime}(\theta(s)) d s, \\
& u(\theta(t))-u\left(t_{k}^{+}\right)=u(\theta(t))-u\left(\theta\left(\theta^{-1}\left(t_{k}\right)^{+}\right)\right)=\int_{\theta^{-1}\left(t_{k}\right)}^{t} u^{\prime}(\theta(s)) d s .
\end{aligned}
$$

Adding these together, we obtain

$$
\begin{aligned}
& u(\theta(t))=u(0)+\int_{0}^{t} u^{\prime}(\theta(s)) d s+\sum_{i=1}^{k}\left[u\left(t_{i}^{+}\right)-u\left(t_{i}\right)\right], \\
& u(\theta(t))=u_{0}+\int_{0}^{t} u^{\prime}(\theta(s)) d s+\sum_{0<\theta^{-1}\left(t_{k}\right)<t} I_{0 k}\left(u\left(t_{k}\right)\right), \quad t \in J .
\end{aligned}
$$

Similarly, we obtain, respectively,

$$
\begin{aligned}
& u^{\prime}(\theta(t))=u_{0}^{\prime}+\int_{0}^{t} u^{\prime \prime}(\theta(s)) d s+\sum_{0<\theta^{-1}\left(t_{k}\right)<t} I_{1 k}\left(u^{\prime}\left(t_{k}\right)\right), \quad t \in J, \\
& u^{\prime \prime}(\theta(t))=u_{0}^{\prime \prime}+\int_{0}^{t} u^{\prime \prime \prime}(\theta(s)) d s+\sum_{0<\theta^{-1}\left(t_{k}\right)<t} I_{2 k}\left(u^{\prime \prime}\left(t_{k}\right)\right), \quad t \in J .
\end{aligned}
$$

Substituting (2.5) into (2.4), it is easy to get

$$
\begin{aligned}
u^{\prime}(\theta(t))= & u_{0}^{\prime}+u_{0}^{\prime \prime} t+\int_{0}^{t}(t-s) u^{\prime \prime \prime}(\theta(s)) d s \\
& +\sum_{0<\theta^{-1}\left(t_{k}\right)<t}\left[I_{1 k}\left(u^{\prime}\left(t_{k}\right)\right)+\left(t-\theta^{-1}\left(t_{k}\right)\right) I_{2 k}\left(u^{\prime \prime}\left(t_{k}\right)\right)\right], \quad t \in J .
\end{aligned}
$$

Substituting (2.6) into (2.3), it is easy to get (2.2).

(ii) Sufficiency.

According to (2.2), it is clear that

$$
u(0)=u_{0}, \quad \Delta u\left(t_{k}\right)=I_{0 k}\left(u\left(t_{k}\right)\right) .
$$

Differentiating both sides of (2.2), we get

$$
\begin{aligned}
u^{\prime}(\theta(t))= & u_{0}^{\prime}+u_{0}^{\prime \prime} t+\int_{0}^{t}(t-s) f\left(s, u(s), u^{\prime}(s), u^{\prime \prime}(s), u\left(\varphi_{1}(s)\right), u\left(\varphi_{2}(s)\right)\right) d s \\
& +\sum_{0<\theta^{-1}\left(t_{k}\right)<t}\left[I_{1 k}\left(u^{\prime}\left(t_{k}\right)\right)+\left(t-\theta^{-1}\left(t_{k}\right)\right) I_{2 k}\left(u^{\prime \prime}\left(t_{k}\right)\right)\right], \quad t \in J .
\end{aligned}
$$

It follows that

$$
u^{\prime}(0)=u_{0}^{\prime}, \quad \Delta u^{\prime}\left(t_{k}\right)=I_{1 k}\left(u^{\prime}\left(t_{k}\right)\right) .
$$

Differentiating both sides of (2.8), we get

$$
\begin{aligned}
u^{\prime \prime}(\theta(t))= & u_{0}^{\prime \prime}+\int_{0}^{t} f\left(s, u(s), u^{\prime}(s), u^{\prime \prime}(s), u\left(\varphi_{1}(s)\right), u\left(\varphi_{2}(s)\right)\right) d s \\
& +\sum_{0<\theta^{-1}\left(t_{k}\right)<t} I_{2 k}\left(u^{\prime \prime}\left(t_{k}\right)\right), \quad t \in J .
\end{aligned}
$$


It follows that

$$
u^{\prime \prime}(0)=u_{0}^{\prime \prime}, \quad \Delta u^{\prime \prime}\left(t_{k}\right)=I_{2 k}\left(u^{\prime \prime}\left(t_{k}\right)\right) \text {. }
$$

Differentiating both sides of (2.10), we get

$$
u^{\prime \prime \prime}(\theta(t))=f\left(t, u(t), u^{\prime}(t), u^{\prime \prime}(t), u\left(\varphi_{1}(t)\right), u\left(\varphi_{2}(t)\right)\right), \quad t \in \bar{J} .
$$

From (2.7), (2.9), (2.11), and (2.12), we see that $u(t)$ is a solution of (1.1).

Lemma 2 (Leray-Schauder [31]) Let the operator $A: X \rightarrow X$ be completely continuous, where $X$ is a real Banach space. If the set

$$
G=\{\|x\| \mid x \in X, x=\lambda A x, 0<\lambda<1\}
$$

is bounded, then the operator $A$ has at least one fixed point in the closed ball

$$
T=\{x \mid x \in X,\|x\| \leq R\}
$$

where $R=\sup G$.

Lemma 3 (Compactness criterion [32]) $H \subset P C(J, \mathbf{R})$ is a relatively compact set if and only if $H \subset P C(J, \mathbf{R})$ is uniformly bounded and equicontinuous on every $J_{k}(k=0, \ldots, p)$, where $J_{0}=\left[t_{0}, t_{1}\right], J_{k}=\left(t_{k}, t_{k+1}\right](k=1, \ldots, p)$.

\section{Main result}

Let us introduce the following conditions for later use:

(H1) There exist nonnegative constants $b_{i}, c_{i k}$ (in the condition, the first three formulas have $i=0,1,2$, the last formulas $i=1,2$, and also $k=1, \ldots, p), d_{1}, d_{2}$, and $g \in L\left(J, \mathbf{R}^{+}\right)$such that

$$
\begin{aligned}
& \left|f\left(t, x_{02}, x_{12}, x_{22}, y_{12}, y_{22}\right)-f\left(t, x_{01}, x_{11}, x_{21}, y_{11}, y_{21}\right)\right| \\
& \quad \leq g(t)\left(\sum_{i=0}^{2} b_{i}\left\|x_{i 2}-x_{i 1}\right\|_{P C}+\sum_{i=1}^{2} d_{i}\left\|y_{i 2}-y_{i 1}\right\|_{P C}\right), \quad t \in J, \\
& \left|I_{i k}\left(x_{i 2}\left(t_{k}\right)\right)-I_{i k}\left(x_{i 1}\left(t_{k}\right)\right)\right| \leq c_{i k}\left|x_{i 2}\left(t_{k}\right)-x_{i 1}\left(t_{k}\right)\right|, \\
& I_{i k}(0)=0,
\end{aligned}
$$

where $x_{01}, x_{02} \in E_{0}, x_{1 i}(t)=\bar{x}_{1 i}^{\prime}(t), x_{2 i}(t)=\bar{x}_{2 i}^{\prime \prime}(t), \bar{x}_{1 i}(t), \bar{x}_{2 i}(t) \in E_{0}, y_{i 1}, y_{i 2} \in P C(J, \mathbf{R})$.

(H2) There exist positive constant $M$ such that

$$
\left|f\left(t, u(t), u^{\prime}(t), u^{\prime \prime}(t), u\left(\varphi_{1}(t)\right), u\left(\varphi_{2}(t)\right)\right)\right| \leq M\left(1+\|u(t)\|_{E_{0}}\right) .
$$

Theorem 1 If conditions (H1), (H2) are satisfied, and 
(H3) $l_{1}, l_{2}, l_{3}<1$, where

$$
\begin{aligned}
& l_{1}=a^{3} M+\sum_{k=1}^{p} \sum_{i=0}^{2} \frac{a^{i}}{i !} c_{i k}, \\
& l_{2}=\frac{m_{12}}{m_{11}}\left[a^{2} M+\sum_{k=1}^{p}\left(c_{1 k}+a c_{2 k}\right)\right], \\
& l_{3}=\frac{m_{12}^{2}}{m_{11}^{3}}\left[m_{11}\left(a M+\sum_{k=1}^{p} c_{2 k}\right)+m_{22}\left(a^{2} M+\sum_{k=1}^{p}\left(c_{1 k}+a c_{2 k}\right)\right)\right],
\end{aligned}
$$

then (1.1) has at least one solution in the closed ball

$$
\bar{B}=\{u(\theta(t)) \mid u(\theta(t)) \in E,\|u(\theta(t))\| \leq R\},
$$

where $R=\sup G$, in which

$$
G=\{\|u(\theta(t))\| \mid u(\theta(t)) \in E, u(\theta(t))=\lambda A u(\theta(t)), 0<\lambda<1\} .
$$

Proof (i) For any $u(\theta(t)) \in E$, define the operator $A$ by

$$
\begin{aligned}
A u(\theta(t))= & \sum_{i=0}^{2} \frac{u_{0}^{(i)}}{i !} t^{i}+\frac{1}{2} \int_{0}^{t}(t-s)^{2} f\left(s, u(s), u^{\prime}(s), u^{\prime \prime}(s), u\left(\varphi_{1}(s)\right), u\left(\varphi_{2}(s)\right)\right) d s \\
& +\sum_{0<\theta^{-1}\left(t_{k}\right)<t} \sum_{i=0}^{2} \frac{\left(t-\theta^{-1}\left(t_{k}\right)\right)^{i}}{i !} I_{i k}\left(u^{(i)}\left(t_{k}\right)\right), \quad t \in J .
\end{aligned}
$$

It is easy to see that $A u(\theta(t)) \in E_{0}$. According to the properties of $\theta$, for any $v(t) \in E_{0}$, we have

$$
\nu(t)=\nu\left(\theta^{-1}(\theta(t))\right)=\nu \theta^{-1}(\theta(t)) .
$$

Let $u=v \theta^{-1}$. Next, it is clear that $v(t)=u(\theta(t)) \in E$. It follows that $A$ maps $E$ into $E$. Thus $A u(\theta(t)) \in E$ with

$$
\begin{aligned}
(A u(\theta(t)))^{\prime}= & u_{0}^{\prime}+u_{0}^{\prime \prime} t+\int_{0}^{t}(t-s) f\left(s, u(s), u^{\prime}(s), u^{\prime \prime}(s), u\left(\varphi_{1}(s)\right), u\left(\varphi_{2}(s)\right)\right) d s \\
& +\sum_{0<\theta^{-1}\left(t_{k}\right)<t}\left[I_{1 k}\left(u^{\prime}\left(t_{k}\right)\right)+\left(t-\theta^{-1}\left(t_{k}\right)\right) I_{2 k}\left(u^{\prime \prime}\left(t_{k}\right)\right)\right], \quad t \in J, \\
(A u(\theta(t)))^{\prime \prime}= & u_{0}^{\prime \prime}+\int_{0}^{t} f\left(s, u(s), u^{\prime}(s), u^{\prime \prime}(s), u\left(\varphi_{1}(s)\right), u\left(\varphi_{2}(s)\right)\right) d s \\
& +\sum_{0<\theta^{-1}\left(t_{k}\right)<t} I_{2 k}\left(u^{\prime \prime}\left(t_{k}\right)\right), \quad t \in J .
\end{aligned}
$$

$A$ is a completely continuous operator, as will be verified by the following three steps. Step $1 . A$ is continuous.

Let any $u_{n}(\theta(t))(n=1,2, \ldots), u(\theta(t)) \in E$ with $\left\|u_{n}(\theta(t))-u(\theta(t))\right\| \rightarrow 0$ as $n \rightarrow \infty$. 
By (3.1) and (H1), we have

$$
\begin{aligned}
\left|A u_{n}(\theta(t))-A u(\theta(t))\right| & \\
\leq & \frac{1}{2} \int_{0}^{t}(t-s)^{2} g(s)\left[\sum_{i=0}^{2} b_{i}\left\|u_{n}^{(i)}(s)-u^{(i)}(s)\right\|_{P C}+\sum_{i=1}^{2} d_{i}\left\|u_{n}\left(\varphi_{i}(s)\right)-u\left(\varphi_{i}(s)\right)\right\|_{P C}\right] d s \\
& +\sum_{0<\theta^{-1}\left(t_{k}\right)<t} \sum_{i=0}^{2} \frac{\left(t-\theta^{-1}\left(t_{k}\right)\right)^{i}}{i !} c_{i k}\left|u_{n}^{(i)}\left(t_{k}\right)-u^{(i)}\left(t_{k}\right)\right| \\
\leq & \left(\sum_{i=0}^{2} b_{i}+\sum_{i=1}^{2} d_{i}\right)\left\|u_{n}(t)-u(t)\right\|_{E_{0}} \int_{0}^{t}(t-s)^{2} g(s) d s \\
& +\left\|u_{n}(t)-u(t)\right\|_{E_{0}} \sum_{0<\theta^{-1}\left(t_{k}\right)<t} \sum_{i=0}^{2} \frac{\left(t-\theta^{-1}\left(t_{k}\right)\right)^{i}}{i !} c_{i k} \\
\leq & {\left[a^{2} a_{0}\left(\sum_{i=0}^{2} b_{i}+\sum_{i=1}^{2} d_{i}\right)+\sum_{k=1}^{p} \sum_{i=0}^{2} \frac{a^{i}}{i !} c_{i k}\right]\left\|u_{n}(t)-u(t)\right\|_{E_{0}}, \quad t \in J }
\end{aligned}
$$

Then, from (3.4) and (2.1), we have

$$
\begin{aligned}
& \left\|A u_{n}(\theta(t))-A u(\theta(t))\right\|_{P C} \\
& \leq\left[a^{2} a_{0}\left(\sum_{i=0}^{2} b_{i}+\sum_{i=1}^{2} d_{i}\right)+\sum_{k=1}^{p} \sum_{i=0}^{2} \frac{a^{i}}{i !} c_{i k}\right]\left\|u_{n}(t)-u(t)\right\|_{E_{0}} \\
& \leq\left[a^{2} a_{0}\left(\sum_{i=0}^{2} b_{i}+\sum_{i=1}^{2} d_{i}\right)+\sum_{k=1}^{p} \sum_{i=0}^{2} \frac{a^{i}}{i !} c_{i k}\right]\left\|u_{n}(\theta(t))-u(\theta(t))\right\| .
\end{aligned}
$$

Thus

$$
\left\|A u_{n}(\theta(t))-A u(\theta(t))\right\|_{P C} \rightarrow 0 \quad \text { as } n \rightarrow \infty .
$$

Similarly, from (3.2) and (2.1), we get

$$
\begin{aligned}
& \left|\frac{d\left[A u_{n}(\theta(t))-A u(\theta(t))\right]}{d \theta(t)}\right| \frac{d \theta}{d t} \\
& \quad=\left|\left(A u_{n}(\theta(t))-A u(\theta(t))\right)^{\prime}\right|=\left|\left(A u_{n}(\theta(t))\right)^{\prime}-(A u(\theta(t)))^{\prime}\right| \\
& \quad \leq\left[a a_{0}\left(\sum_{i=0}^{2} b_{i}+\sum_{i=1}^{2} d_{i}\right)+\sum_{k=1}^{p}\left(c_{1 k}+a c_{2 k}\right)\right]\left\|u_{n}(\theta(t))-u(\theta(t))\right\|, \\
& \left|\frac{d\left[A u_{n}(\theta(t))-A u(\theta(t))\right]}{d \theta(t)}\right| \\
& \quad \leq \frac{1}{m_{11}}\left[a a_{0}\left(\sum_{i=0}^{2} b_{i}+\sum_{i=1}^{2} d_{i}\right)+\sum_{k=1}^{p}\left(c_{1 k}+a c_{2 k}\right)\right]\left\|u_{n}(\theta(t))-u(\theta(t))\right\|, \quad t \in J, \\
& \left\|\left(A u_{n}(\theta(t))-A u(\theta(t))\right)^{\prime}\right\|_{P C^{*}} \\
& \quad \leq \frac{m_{12}}{m_{11}}\left[a a_{0}\left(\sum_{i=0}^{2} b_{i}+\sum_{i=1}^{2} d_{i}\right)+\sum_{k=1}^{p}\left(c_{1 k}+a c_{2 k}\right)\right]\left\|u_{n}(\theta(t))-u(\theta(t))\right\| .
\end{aligned}
$$


Thus

$$
\left\|\left(A u_{n}(\theta(t))-A u(\theta(t))\right)^{\prime}\right\|_{P C^{*}} \rightarrow 0 \quad \text { as } n \rightarrow \infty .
$$

Similarly, from (3.3) and (2.1), we get

$$
\begin{aligned}
& \left|\frac{d^{2}\left[A u_{n}(\theta(t))-A u(\theta(t))\right]}{d \theta^{2}(t)} \cdot\left(\frac{d \theta}{d t}\right)^{2}+\frac{d\left[A u_{n}(\theta(t))-A u(\theta(t))\right]}{d \theta(t)} \cdot \frac{d^{2} \theta}{d t^{2}}\right| \\
& \quad=\left|\left(A u_{n}(\theta(t))-A u(\theta(t))\right)^{\prime \prime}\right|=\left|\left(A u_{n}(\theta(t))\right)^{\prime \prime}-(A u(\theta(t)))^{\prime \prime}\right| \\
& \quad \leq\left[a_{0}\left(\sum_{i=0}^{2} b_{i}+\sum_{i=1}^{2} d_{i}\right)+\sum_{k=1}^{p} c_{2 k}\right]\left\|u_{n}(\theta(t))-u(\theta(t))\right\|,
\end{aligned}
$$

by (3.7) and the properties of $\theta$, it follows that

$$
\begin{aligned}
& \mid \frac{d^{2}\left[A u_{n}(\theta(t))-A u(\theta(t))\right]}{d \theta^{2}(t)} \mid \cdot\left(\frac{d \theta}{d t}\right)^{2} \\
&=\left|\frac{d^{2}\left[A u_{n}(\theta(t))-A u(\theta(t))\right]}{d \theta^{2}(t)} \cdot\left(\frac{d \theta}{d t}\right)^{2}\right| \\
& \leq\left|\frac{d^{2}\left[A u_{n}(\theta(t))-A u(\theta(t))\right]}{d \theta^{2}(t)} \cdot\left(\frac{d \theta}{d t}\right)^{2}+\frac{d\left[A u_{n}(\theta(t))-A u(\theta(t))\right]}{d \theta(t)} \cdot \frac{d^{2} \theta}{d t^{2}}\right| \\
&+\left|\frac{d\left[A u_{n}(\theta(t))-A u(\theta(t))\right]}{d \theta(t)}\right| \cdot\left|\frac{d^{2} \theta}{d t^{2}}\right| \\
& \leq {\left[a_{0}\left(\sum_{i=0}^{2} b_{i}+\sum_{i=1}^{2} d_{i}\right)+\sum_{k=1}^{p} c_{2 k}\right]\left\|u_{n}(\theta(t))-u(\theta(t))\right\| } \\
&+\frac{m_{22}}{m_{11}}\left[a a_{0}\left(\sum_{i=0}^{2} b_{i}+\sum_{i=1}^{2} d_{i}\right)+\sum_{k=1}^{p}\left(c_{1 k}+a c_{2 k}\right)\right]\left\|u_{n}(\theta(t))-u(\theta(t))\right\| \\
& \leq \frac{d^{2}\left[A u_{n}(\theta(t))-A u(\theta(t))\right]}{d \theta^{2}(t)} \\
& \leq \times\left\|u_{n}(\theta(t))-u(\theta(t))\right\|, t \in J, \\
& m_{11}^{3}\left\{a_{0}\left(\sum_{i=0}^{2} b_{i}+\sum_{i=1}^{2} d_{i}\right)\left(m_{11}+a m_{22}\right)+\sum_{k=1}^{p}\left[m_{22} c_{1 k}+\left(m_{11}+a m_{22}\right) c_{2 k}\right]\right\}
\end{aligned}
$$

so

$$
\begin{aligned}
\left\|\left(A u_{n}(\theta(t))-A u(\theta(t))\right)^{\prime \prime}\right\|_{P C^{* *}} \\
\leq \frac{m_{12}^{2}}{m_{11}^{3}}\left\{a_{0}\left(\sum_{i=0}^{2} b_{i}+\sum_{i=1}^{2} d_{i}\right)\left(m_{11}+a m_{22}\right)+\sum_{k=1}^{p}\left[m_{22} c_{1 k}+\left(m_{11}+a m_{22}\right) c_{2 k}\right]\right\} \\
\quad \times\left\|u_{n}(\theta(t))-u(\theta(t))\right\| .
\end{aligned}
$$

Thus

$$
\left\|\left(A u_{n}(\theta(t))-A u(\theta(t))\right)^{\prime \prime}\right\|_{P C^{* *}} \rightarrow 0 \quad \text { as } n \rightarrow \infty .
$$


By (3.6), (3.9), and (3.11), it is easy to see that $\left\|A u_{n}(\theta(t))-A u(\theta(t))\right\| \rightarrow 0$ as $n \rightarrow \infty$, that is to say, $A$ is continuous.

Step 2. $A$ maps any bounded subset of $E$ into one bounded subset of $E$.

Let $T$ be any bounded subset of $E$. Then there exists $h>0$ such that $\|u(\theta(t))\| \leq h$ for all $u(\theta(t)) \in T$.

By (3.1), (H1), (H2), and (2.1), we have

$$
\begin{aligned}
|A u(\theta(t))| \leq & \sum_{i=0}^{2} \frac{\left|u_{0}^{(i)}\right|}{i !} t^{i}+\frac{1}{2} \int_{0}^{t}(t-s)^{2} M\left(1+\|u(s)\|_{E_{0}}\right) d s \\
& +\sum_{0<\theta^{-1}\left(t_{k}\right)<t} \sum_{i=0}^{2} \frac{\left(t-\theta^{-1}\left(t_{k}\right)\right)^{i}}{i !} c_{i k}\left|u^{(i)}\left(t_{k}\right)\right| \\
\leq & \sum_{i=0}^{2} \frac{a^{i}}{i !}\left|u_{0}^{(i)}\right|+M\left(1+\|u(t)\|_{E_{0}}\right) \int_{0}^{a} a^{2} d s+\|u(t)\|_{E_{0}} \sum_{0<\theta^{-1}\left(t_{k}\right)<t} \sum_{i=0}^{2} \frac{a^{i}}{i !} c_{i k} \\
\leq & \sum_{i=0}^{2} \frac{a^{i}}{i !}\left|u_{0}^{(i)}\right|+a^{3} M(1+\|u(\theta(t))\|)+\sum_{k=1}^{p} \sum_{i=0}^{2} \frac{a^{i}}{i !} c_{i k}\|u(\theta(t))\| \\
\leq & \sum_{i=0}^{2} \frac{a^{i}}{i !}\left|u_{0}^{(i)}\right|+a^{3} M(1+h)+h \sum_{k=1}^{p} \sum_{i=0}^{2} \frac{a^{i}}{i !} c_{i k}, \quad t \in J,
\end{aligned}
$$

So

$$
\|A u(\theta(t))\|_{P C} \leq \sum_{i=0}^{2} \frac{a^{i}}{i !}\left|u_{0}^{(i)}\right|+a^{3} M(1+h)+h \sum_{k=1}^{p} \sum_{i=0}^{2} \frac{a^{i}}{i !} c_{i k} .
$$

Similarly, from (3.2), (H1), (H2), and (2.1), we get

$$
\begin{aligned}
& \left|\frac{d A u(\theta(t))}{d \theta(t)}\right| \cdot \frac{d \theta}{d t}=\left|(A u(\theta(t)))^{\prime}\right| \\
& \leq\left|u_{0}^{\prime}\right|+a\left|u_{0}^{\prime \prime}\right|+a^{2} M(1+h)+h \sum_{k=1}^{p}\left(c_{1 k}+a c_{2 k}\right), \quad t \in J \\
& \left|\frac{d A u(\theta(t))}{d \theta(t)}\right| \leq \frac{1}{m_{11}}\left[\left|u_{0}^{\prime}\right|+a\left|u_{0}^{\prime \prime}\right|+a^{2} M(1+h)+h \sum_{k=1}^{p}\left(c_{1 k}+a c_{2 k}\right)\right], \quad t \in J,
\end{aligned}
$$

so

$$
\left\|(A u(\theta(t)))^{\prime}\right\|_{P C^{*}} \leq \frac{m_{12}}{m_{11}}\left[\left|u_{0}^{\prime}\right|+a\left|u_{0}^{\prime \prime}\right|+a^{2} M(1+h)+h \sum_{k=1}^{p}\left(c_{1 k}+a c_{2 k}\right)\right]
$$

Similarly, from (3.3), (H1), (H2), (3.13), (2.1), and the properties of $\theta$, we get

$$
\begin{aligned}
& \left|\frac{d^{2} A u(\theta(t))}{d \theta^{2}(t)}\right| \cdot\left(\frac{d \theta}{d t}\right)^{2} \\
& =\left|\frac{d^{2} A u(\theta(t))}{d \theta^{2}(t)} \cdot\left(\frac{d \theta}{d t}\right)^{2}\right|
\end{aligned}
$$




$$
\begin{aligned}
& \leq\left|\frac{d^{2} A u(\theta(t))}{d \theta^{2}(t)} \cdot\left(\frac{d \theta}{d t}\right)^{2}+\frac{d A u(\theta(t))}{d \theta(t)} \cdot \frac{d^{2} \theta}{d t^{2}}\right|+\left|\frac{d A u(\theta(t))}{d \theta(t)}\right| \cdot\left|\frac{d^{2} \theta}{d t^{2}}\right| \\
& =\left|(A u(\theta(t)))^{\prime \prime}\right|+\left|\frac{d A u(\theta(t))}{d \theta(t)}\right| \cdot\left|\frac{d^{2} \theta}{d t^{2}}\right| \\
& \leq \quad\left[\left|u_{0}^{\prime \prime}\right|+a M(1+h)+h \sum_{k=1}^{p} c_{2 k}\right] \\
& \quad+\frac{m_{22}}{m_{11}}\left[\left|u_{0}^{\prime}\right|+a\left|u_{0}^{\prime \prime}\right|+a^{2} M(1+h)+h \sum_{k=1}^{p}\left(c_{1 k}+a c_{2 k}\right)\right], \\
& \left|\frac{d^{2} A u(\theta(t))}{d \theta^{2}(t)}\right| \leq \frac{1}{m_{11}^{2}}\left[\left|u_{0}^{\prime \prime}\right|+a M(1+h)+h \sum_{k=1}^{p} c_{2 k}\right] \\
& \quad+\frac{m_{22}}{m_{11}^{3}}\left[\left|u_{0}^{\prime}\right|+a\left|u_{0}^{\prime \prime}\right|+a^{2} M(1+h)+h \sum_{k=1}^{p}\left(c_{1 k}+a c_{2 k}\right)\right]
\end{aligned}
$$

so

$$
\begin{aligned}
\left\|(A u(\theta(t)))^{\prime \prime}\right\|_{P C^{* *}} \leq & \frac{m_{12}^{2}}{m_{11}^{3}}\left\{m_{11}\left[\left|u_{0}^{\prime \prime}\right|+a M(1+h)+h \sum_{k=1}^{p} c_{2 k}\right]\right. \\
& \left.+m_{22}\left[\left|u_{0}^{\prime}\right|+a\left|u_{0}^{\prime \prime}\right|+a^{2} M(1+h)+h \sum_{k=1}^{p}\left(c_{1 k}+a c_{2 k}\right)\right]\right\} .
\end{aligned}
$$

According to (3.12), (3.14), and (3.15), we obtain

$$
\begin{aligned}
\|A u(\theta(t))\| \leq & \max \left\{\frac{a^{i}}{i !}\left|u_{0}^{(i)}\right|+a^{3} M(1+h)+h \sum_{k=1}^{p} \sum_{i=0}^{2} \frac{a^{i}}{i !} c_{i k},\right. \\
& \frac{m_{12}}{m_{11}}\left[\left|u_{0}^{\prime}\right|+a\left|u_{0}^{\prime \prime}\right|+a^{2} M(1+h)+h \sum_{k=1}^{p}\left(c_{1 k}+a c_{2 k}\right)\right], \\
& \frac{m_{12}^{2}}{m_{11}^{3}}\left\{m_{11}\left[\left|u_{0}^{\prime \prime}\right|+a M(1+h)+h \sum_{k=1}^{p} c_{2 k}\right]\right. \\
& \left.+m_{22}\left[\left|u_{0}^{\prime}\right|+a\left|u_{0}^{\prime \prime}\right|+a^{2} M(1+h)+h \sum_{k=1}^{p}\left(c_{1 k}+a c_{2 k}\right)\right]\right\} .
\end{aligned}
$$

Therefore, $A(T)$ is uniformly bounded.

Step 3. $A(T)$ is equicontinuous on every $J_{k}(k=0, \ldots, p)$, where $J_{0}=\left[0, \theta^{-1}\left(t_{1}\right)\right], J_{k}=$ $\left(\theta^{-1}\left(t_{k}\right), \theta^{-1}\left(t_{k+1}\right)\right](k=1, \ldots, p)$.

For any $A u(\theta(t)) \in A(T)$ and any $\varepsilon>0$, take

$$
\delta=\left[\left|u_{0}^{\prime}\right|+a\left|u_{0}^{\prime \prime}\right|+a^{2} M(1+h)+h \sum_{i=1}^{k}\left(c_{1 i}+a c_{2 i}\right)\right]^{-1} \varepsilon .
$$

Then if $t_{1}, t_{2} \in J_{k}$ and $\left|t_{1}-t_{2}\right|<\delta$ with $t_{1}<t_{2}$, from (3.1), (H1), (H2), and (2.1), we have

$$
\begin{aligned}
& \left|A u\left(\theta\left(t_{2}\right)\right)-A u\left(\theta\left(t_{1}\right)\right)\right| \\
& \quad \leq\left|u_{0}^{\prime}\right|\left(t_{2}-t_{1}\right)+\frac{1}{2}\left|u_{0}^{\prime \prime}\right|\left(t_{2}+t_{1}\right)\left(t_{2}-t_{1}\right)+\frac{1}{2} \int_{t_{1}}^{t_{2}}(t-s)^{2} M\left(1+\|u(s)\|_{E_{0}}\right) d s
\end{aligned}
$$




$$
\begin{aligned}
& +\sum_{i=1}^{k}\left[\left(t_{2}-t_{1}\right) c_{1 i}\left|u^{\prime}\left(t_{i}\right)\right|+\frac{1}{2}\left|\left(t_{2}-\theta^{-1}\left(t_{i}\right)\right)+\left(t_{1}-\theta^{-1}\left(t_{i}\right)\right)\right|\left(t_{2}-t_{1}\right) c_{2 i}\left|u^{\prime \prime}\left(t_{i}\right)\right|\right] \\
\leq & {\left[\left|u_{0}^{\prime}\right|+a\left|u_{0}^{\prime \prime}\right|+a^{2} M\left(1+\|u(t)\|_{E_{0}}\right)+\|u(t)\|_{E_{0}} \sum_{i=1}^{k}\left(c_{1 i}+a c_{2 i}\right)\right]\left(t_{2}-t_{1}\right) } \\
\leq & {\left[\left|u_{0}^{\prime}\right|+a\left|u_{0}^{\prime \prime}\right|+a^{2} M(1+\|u(\theta(t))\|)+\|u(\theta(t))\| \sum_{i=1}^{k}\left(c_{1 i}+a c_{2 i}\right)\right]\left|t_{2}-t_{1}\right| } \\
\leq & {\left[\left|u_{0}^{\prime}\right|+a\left|u_{0}^{\prime \prime}\right|+a^{2} M(1+h)+h \sum_{i=1}^{k}\left(c_{1 i}+a c_{2 i}\right)\right]\left|t_{2}-t_{1}\right|<\varepsilon . }
\end{aligned}
$$

Thus, $A(T)$ is equicontinuous on every $J_{k}(k=0, \ldots, p)$.

As a consequence of Steps 1-3, $A$ is completely continuous.

(ii) For any $\|u(\theta(t))\| \in G$, similar to getting (3.12), (3.14), and (3.15), we have, respectively,

$$
\begin{aligned}
\|A u(\theta(t))\|_{P C} \leq & \sum_{i=0}^{2} \frac{a^{i}}{i !}\left|u_{0}^{(i)}\right|+a^{3} M+\left[a^{3} M+\sum_{k=1}^{p} \sum_{i=0}^{2} \frac{a^{i}}{i !} c_{i k}\right]\|u(\theta(t))\| \\
=\sum_{i=0}^{2} & \frac{a^{i}}{i !}\left|u_{0}^{(i)}\right|+a^{3} M+l_{1}\|u(\theta(t))\|, \\
\left\|(A u(\theta(t)))^{\prime}\right\|_{P C^{*}} \leq & \frac{m_{12}}{m_{11}}\left(\left|u_{0}^{\prime}\right|+a\left|u_{0}^{\prime \prime}\right|+a^{2} M\right)+\frac{m_{12}}{m_{11}}\left[a^{2} M+\sum_{k=1}^{p}\left(c_{1 k}+a c_{2 k}\right)\right]\|u(\theta(t))\| \\
= & \frac{m_{12}}{m_{11}}\left(\left|u_{0}^{\prime}\right|+a\left|u_{0}^{\prime \prime}\right|+a^{2} M\right)+l_{2}\|u(\theta(t))\|, \\
\left\|(A u(\theta(t)))^{\prime \prime}\right\|_{P C^{* *}} \leq & \frac{m_{12}^{2}}{m_{11}^{3}}\left\{\left[m_{11}\left(\left|u_{0}^{\prime \prime}\right|+a M\right)+m_{22}\left(\left|u_{0}^{\prime}\right|+a\left|u_{0}^{\prime \prime}\right|+a^{2} M\right)\right]\right. \\
& \left.+\left[m_{11}\left(a M+\sum_{k=1}^{p} c_{2 k}\right)+m_{22}\left(a M^{2}+\sum_{k=1}^{p}\left(c_{1 k}+a c_{2 k}\right)\right)\right]\right\} \\
& \times\|u(\theta(t))\| \\
= & \frac{m_{12}^{2}}{m_{11}^{3}}\left[m_{11}\left(\left|u_{0}^{\prime \prime}\right|+a M\right)+m_{22}\left(\left|u_{0}^{\prime}\right|+a\left|u_{0}^{\prime \prime}\right|+a^{2} M\right)\right]+l_{3}\|u(\theta(t))\| .
\end{aligned}
$$

Then

$$
\|u(\theta(t))\|=\lambda\|A u(\theta(t))\| \leq\|A u(\theta(t))\| \leq L+l\|u(\theta(t))\|,
$$

where

$$
\begin{aligned}
L= & \max \left\{\sum_{i=0}^{2} \frac{a^{i}}{i !}\left|u_{0}^{(i)}\right|+a^{3} M, \frac{m_{12}}{m_{11}}\left(\left|u_{0}^{\prime}\right|+a\left|u_{0}^{\prime \prime}\right|+a^{2} M\right),\right. \\
& \left.\frac{m_{12}^{2}}{m_{11}^{3}}\left[m_{11}\left(\left|u_{0}^{\prime \prime}\right|+a M\right)+m_{22}\left(\left|u_{0}^{\prime}\right|+a\left|u_{0}^{\prime \prime}\right|+a^{2} M\right)\right]\right\}
\end{aligned}
$$

and $l=\max \left\{l_{1}, l_{2}, l_{3}\right\}$. 
It follows that $\|u(\theta(t))\| \leq \frac{L}{1-l}$, i.e., $G$ is bounded.

From (i) and (ii), all conditions of Lemma 2 are satisfied. Therefore, the proof is complete.

Theorem 2 If condition $(\mathrm{H} 1)\left(I_{i k}(0)=0\right.$ are not needed $)$ is satisfied, and

(H4) $r_{1}, r_{2}, r_{3}<1$, where

$$
\begin{aligned}
r_{1}= & a^{2} a_{0}\left(\sum_{i=0}^{2} b_{i}+\sum_{i=1}^{2} d_{i}\right)+\sum_{k=1}^{p} \sum_{i=0}^{2} \frac{a^{i}}{i !} c_{i k}, \\
r_{2}= & \frac{m_{12}}{m_{11}}\left[a a_{0}\left(\sum_{i=0}^{2} b_{i}+\sum_{i=1}^{2} d_{i}\right)+\sum_{k=1}^{p}\left(c_{1 k}+a c_{2 k}\right)\right], \\
r_{3}= & \frac{m_{12}^{2}}{m_{11}^{3}}\left\{a_{0}\left(\sum_{i=0}^{2} b_{i}+\sum_{i=1}^{2} d_{i}\right)\left(m_{11}+a m_{22}\right)\right. \\
& \left.+\sum_{k=1}^{p}\left[m_{22} c_{1 k}+\left(m_{11}+a m_{22}\right) c_{2 k}\right]\right\}, \quad \text { in which } a_{0}=\int_{0}^{a} g(t) d t,
\end{aligned}
$$

then (1.1) has a unique solution.

Proof Similar with getting (3.5), (3.8) and (3.10), for any $u_{1}(\theta(t)), u_{2}(\theta(t)) \in E$, we obtain, respectively,

$$
\begin{aligned}
\| & A u_{2}(\theta(t))-A u_{1}(\theta(t)) \|_{P C} \\
\leq & {\left[a^{2} a_{0}\left(\sum_{i=0}^{2} b_{i}+\sum_{i=1}^{2} d_{i}\right)+\sum_{k=1}^{p} \sum_{i=0}^{2} \frac{a^{i}}{i !} c_{i k}\right]\left\|u_{2}(\theta(t))-u_{1}(\theta(t))\right\| } \\
= & r_{1}\left\|u_{2}(\theta(t))-u_{1}(\theta(t))\right\|, \\
\| & \left(A u_{2}(\theta(t))-A u_{1}(\theta(t))\right)^{\prime} \|_{P C^{*}} \\
\leq & \frac{m_{12}}{m_{11}}\left[a a_{0}\left(\sum_{i=0}^{2} b_{i}+\sum_{i=1}^{2} d_{i}\right)+\sum_{k=1}^{p}\left(c_{1 k}+a c_{2 k}\right)\right]\left\|u_{2}(\theta(t))-u_{1}(\theta(t))\right\| \\
= & r_{2}\left\|u_{2}(\theta(t))-u_{1}(\theta(t))\right\|, \\
\left\|\left(A u_{2}(\theta(t))-A u_{1}(\theta(t))\right)^{\prime \prime}\right\|_{P C^{* *}} & m_{12}^{2}\left\{a_{0}\left(\sum_{i=0}^{2} b_{i}+\sum_{i=1}^{2} d_{i}\right)\left(m_{11}+a m_{22}\right)\right. \\
m_{11}^{3} & \left.+\sum_{k=1}^{p}\left[m_{22} c_{1 k}+\left(m_{11}+a m_{22} c_{2 k}\right)\right]\right\}\left\|u_{2}(\theta(t))-u_{1}(\theta(t))\right\| \\
= & r_{3}\left\|u_{2}(\theta(t))-u_{1}(\theta(t))\right\| .
\end{aligned}
$$

Then

$$
\left\|A u_{2}(\theta(t))-A u_{1}(\theta(t))\right\| \leq \max \left\{r_{1}, r_{2}, r_{3}\right\}\left\|u_{2}(\theta(t))-u_{1}(\theta(t))\right\| .
$$


Thus all conditions of the fixed-point theorem of Banach are satisfied. The proof is complete.

Remark 1 By comparing Theorem 1 with Theorem 2, it is easy to see that each of them has its own strong and weak points. The condition (H3) of Theorem 1 is satisfied more easily than the condition (H4) of Theorem 2. The condition (H2) of Theorem 1 is also satisfied easily, but it does not need in Theorem 2 .

\section{Examples}

Example 1 Consider the equation

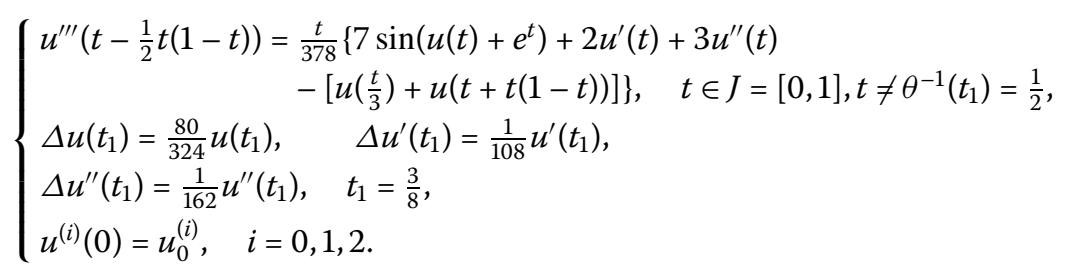

Firstly, it is easy to verify that

$$
\theta(t)=t-\frac{1}{2} t(1-t), \quad \varphi_{1}(t)=\frac{t}{3}, \quad \varphi_{2}(t)=t+t(1-t)
$$

all satisfy the requisitions of (1.1). From $\theta^{\prime}(t)=\frac{1}{2}+t$ and $\theta^{\prime \prime}(t)=1$, we get that

$$
m_{11}=1 / 2, \quad m_{12}=3 / 2, \quad m_{21}=m_{22}=1 .
$$

Next, since

$$
f\left(t, x_{0}, x_{1}, x_{2}, y_{1}, y_{2}\right)=\frac{t}{378}\left[7 \sin \left(x_{0}+e^{t}\right)+2 x_{1}+3 x_{2}-\left(y_{1}+y_{2}\right)\right]
$$

and

$$
\begin{aligned}
& \left|\sin \left(x_{02}(t)+e^{t}\right)-\sin \left(x_{01}(t)+e^{t}\right)\right| \\
& \quad=\left|\left(x_{02}(t)+e^{t}\right)-\left(x_{01}(t)+e^{t}\right)\right| \cdot\left|\cos \left(\bar{x}_{0}(t)+e^{t}\right)\right| \leq\left|x_{02}(t)-x_{01}(t)\right|,
\end{aligned}
$$

where $\bar{x}_{0}(t)$ is located between $x_{02}(t)$ and $x_{01}(t)$, we have

$$
\begin{aligned}
& \left|f\left(t, x_{02}, x_{12}, x_{22}, y_{12}, y_{22}\right)-f\left(t, x_{01}, x_{11}, x_{21}, y_{11}, y_{21}\right)\right| \\
& \leq \frac{t}{378}\left[7\left|\sin \left(x_{02}+e^{t}\right)-\sin \left(x_{01}+e^{t}\right)\right|+2\left|x_{12}-x_{11}\right|\right. \\
& \left.\quad+3\left|x_{22}-x_{21}\right|+\sum_{i=1}^{2}\left|y_{i 2}-y_{i 1}\right|\right] \\
& \leq t\left[\frac{1}{54}\left|x_{02}-x_{01}\right|+\frac{1}{189}\left|x_{12}-x_{11}\right|+\frac{1}{126}\left|x_{22}-x_{21}\right|+\frac{1}{378} \sum_{i=1}^{2}\left|y_{i 2}-y_{i 1}\right|\right]
\end{aligned}
$$




$$
\begin{aligned}
\leq & t\left[\frac{1}{54}\left\|x_{02}-x_{01}\right\|_{P C}+\frac{1}{189}\left\|x_{12}-x_{11}\right\|_{P C}+\frac{1}{126}\left\|x_{22}-x_{21}\right\|_{P C}\right. \\
& \left.+\frac{1}{378} \sum_{i=1}^{2}\left\|y_{i 2}-y_{i 1}\right\|_{P C}\right], \quad t \in J
\end{aligned}
$$

where $b_{0}=\frac{1}{54}, b_{1}=\frac{1}{189}, b_{2}=\frac{1}{126}, d_{1}=d_{2}=\frac{1}{378}, a=1, a_{0}=\int_{0}^{1} t d t=\frac{1}{2}$. From $I_{01}\left(x_{0}\right)=\frac{80}{324} x_{0}$, $I_{11}\left(x_{1}\right)=\frac{1}{108} x_{1}, I_{21}\left(x_{2}\right)=\frac{1}{162} x_{2}$, we have

$$
\begin{aligned}
& \left|I_{01}\left(x_{02}\left(t_{1}\right)\right)-I_{01}\left(x_{01}\left(t_{1}\right)\right)\right| \leq \frac{80}{324}\left|x_{02}\left(t_{1}\right)-x_{01}\left(t_{1}\right)\right|, \quad I_{01}(0)=0, \\
& \left|I_{11}\left(x_{12}\left(t_{1}\right)\right)-I_{11}\left(x_{11}\left(t_{1}\right)\right)\right| \leq \frac{1}{108}\left|x_{12}\left(t_{1}\right)-x_{11}\left(t_{1}\right)\right|, \quad I_{11}(0)=0, \\
& \left|I_{21}\left(x_{22}\left(t_{1}\right)\right)-I_{21}\left(x_{21}\left(t_{1}\right)\right)\right| \leq \frac{1}{162}\left|x_{22}\left(t_{1}\right)-x_{21}\left(t_{1}\right)\right|, \quad I_{21}(0)=0,
\end{aligned}
$$

where $c_{01}=\frac{80}{324}, c_{11}=\frac{1}{108}, c_{21}=\frac{1}{162}$. Further, we have

$$
\begin{aligned}
& \left|f\left(t, u(t), u^{\prime}(t), u^{\prime \prime}(t), u\left(\frac{t}{3}\right), u(t+t(1-t))\right)\right| \\
& \quad \leq \frac{1}{378}\left\{7\left|\sin \left(u(t)+e^{t}\right)\right|+2\left|u^{\prime}(t)\right|+3\left|u^{\prime \prime}(t)\right|+\left[\left|u\left(\frac{t}{3}\right)\right|+|u(t+t(1-t))|\right]\right\} \\
& \quad \leq \frac{1}{378}\left[7+2\|u(t)\|_{E_{0}}+3\|u(t)\|_{E_{0}}+\left(\|u(t)\|_{E_{0}}+\|u(t)\|_{E_{0}}\right)\right] \\
& \quad=\frac{1}{54}\left(1+\|u(t)\|_{E_{0}}\right),
\end{aligned}
$$

where $M=\frac{1}{54}$.

Finally, we get

$$
\begin{aligned}
& l_{1}=M+c_{01}+c_{11}+\frac{1}{2} c_{21}=\frac{5}{18}<1, \\
& l_{2}=3\left(M+c_{11}+c_{21}\right)=\frac{11}{108}<1, \\
& l_{3}=9\left(3 M+2 c_{11}+3 c_{21}\right)=\frac{5}{6}<1 .
\end{aligned}
$$

Thus, (4.1) satisfies all conditions of Theorem 1. It follows that (4.1) has at least one solution in the closed ball $\bar{B}$.

Example 2 Consider the equation

$$
\left\{\begin{aligned}
& u^{\prime \prime \prime}\left(t-\frac{1}{2} t(1-t)\right)= \frac{t}{432}\left\{2 \sqrt{1+u^{2}(t)}-2 \arctan \left(u^{\prime}(t)+e^{t}\right)\right. \\
&\left.+6 u^{\prime \prime}(t)-3\left[u\left(\frac{t}{2}\right)+u\left(t^{3}\right)\right]\right\}, \quad t \in J=[0,1], t \neq \theta^{-1}\left(t_{1}\right)=\frac{1}{2}, \\
& \Delta u\left(t_{1}\right)=\frac{8}{324} u\left(t_{1}\right)+1, \quad \Delta u^{\prime}\left(t_{1}\right)=\frac{1}{108} u^{\prime}\left(t_{1}\right)+2, \\
& \Delta u^{\prime \prime}\left(t_{1}\right)=\frac{1}{162} u^{\prime \prime}\left(t_{1}\right)+3, \quad t_{1}=\frac{3}{8}, \\
& u^{(i)}(0)=u_{0}^{(i)}, \quad i=0,1,2 .
\end{aligned}\right.
$$

Firstly, it is easy to verify that

$$
\theta(t)=t-\frac{1}{2} t(1-t), \quad \varphi_{1}(t)=\frac{t}{2}, \quad \varphi_{2}(t)=t^{3}
$$


all satisfy the requisitions of (1.1). From $\theta^{\prime}(t)=\frac{1}{2}+t$ and $\theta^{\prime \prime}(t)=1$, we get

$$
m_{1}=1 / 2, \quad m_{2}=3 / 2, \quad m_{21}=m_{22}=1 .
$$

Next, since

$$
f\left(t, x_{0}, x_{1}, x_{2}, y_{1}, y_{2}\right)=\frac{t}{432}\left[2 \sqrt{1+x_{0}^{2}}-2 \arctan \left(x_{1}+e^{t}\right)+6 x_{2}-3\left(y_{1}+y_{2}\right)\right]
$$

and

$$
\begin{aligned}
& \left|\sqrt{1+x_{02}^{2}(t)}-\sqrt{1+x_{01}^{2}(t)}\right| \\
& \quad=\left|x_{02}(t)-x_{01}(t)\right| \cdot \frac{\left|\bar{x}_{0}(t)\right|}{1+\left|\bar{x}_{0}(t)\right|^{2}} \leq\left|x_{02}(t)-x_{01}(t)\right|, \\
& \quad=\left|\left(x_{12}(t)+e^{t}\right)-\left(x_{11}(t)+e^{t}\right)\right| \cdot \frac{1}{1+\left(\bar{x}_{1}(t)+e^{t}\right)^{2}} \leq\left|x_{12}(t)-x_{11}(t)\right|,
\end{aligned}
$$

where $\bar{x}_{0}(t)$ is located between $x_{02}(t)$ and $x_{01}(t), \bar{x}_{1}(t)$ is located between $x_{12}(t)$ and $x_{11}(t)$, we have

$$
\begin{aligned}
\mid f( & \left.t, x_{02}, x_{12}, x_{22}, y_{12}, y_{22}\right)-f\left(t, x_{01}, x_{11}, x_{21}, y_{11}, y_{21}\right) \mid \\
\leq & \frac{t}{432}\left[2\left|\sqrt{1+x_{02}^{2}}-\sqrt{1+x_{01}^{2}}\right|+2\left|\arctan \left(x_{12}+e^{t}\right)-\arctan \left(x_{11}+e^{t}\right)\right|\right. \\
& \left.+6\left|x_{22}-x_{21}\right|+\sum_{i=1}^{2}\left|y_{i 2}-y_{i 1}\right|\right] \\
\leq & t\left[\frac{1}{216}\left|x_{02}-x_{01}\right|+\frac{1}{216}\left|x_{12}-x_{11}\right|+\frac{1}{72}\left|x_{22}-x_{21}\right|+\frac{3}{432} \sum_{i=1}^{2}\left|y_{i 2}-y_{i 1}\right|\right] \\
\leq & t\left[\frac{1}{216}\left\|x_{02}-x_{01}\right\|_{P C}+\frac{1}{216}\left\|x_{12}-x_{11}\right\|_{P C}+\frac{1}{72}\left\|x_{22}-x_{21}\right\|_{P C}\right. \\
& \left.+\frac{3}{432} \sum_{i=1}^{2}\left\|y_{i 2}-y_{i 1}\right\|_{P C}\right], \quad t \in J
\end{aligned}
$$

where $b_{0}=b_{1}=\frac{1}{216}, b_{2}=\frac{1}{72}, d_{1}=d_{2}=\frac{1}{432}, a=1, a_{0}=\int_{0}^{1} t d t=\frac{1}{2}$. From $I_{01}\left(x_{0}\right)=\frac{8}{324} x_{0}+1$, $I_{11}\left(x_{1}\right)=\frac{1}{108} x_{1}+2, I_{21}\left(x_{1}\right)=\frac{1}{162} x_{2}+3$, we have

$$
\begin{aligned}
& \left|I_{01}\left(x_{02}\left(t_{1}\right)\right)-I_{01}\left(x_{01}\left(t_{1}\right)\right)\right| \leq \frac{8}{324}\left|x_{02}\left(t_{1}\right)-x_{01}\left(t_{1}\right)\right|, \\
& \left|I_{11}\left(x_{12}\left(t_{1}\right)\right)-I_{11}\left(x_{11}\left(t_{1}\right)\right)\right| \leq \frac{1}{108}\left|x_{12}\left(t_{1}\right)-x_{11}\left(t_{1}\right)\right|, \\
& \left|I_{21}\left(x_{22}\left(t_{1}\right)\right)-I_{21}\left(x_{21}\left(t_{1}\right)\right)\right| \leq \frac{1}{162}\left|x_{22}\left(t_{1}\right)-x_{21}\left(t_{1}\right)\right|,
\end{aligned}
$$

where $c_{01}=\frac{8}{324}, c_{11}=\frac{1}{108}, c_{21}=\frac{1}{162}$. 
Finally, we get

$$
\begin{aligned}
& r_{1}=\frac{1}{2}\left(\sum_{i=0}^{2} b_{i}+\sum_{i=1}^{2} d_{i}\right)+c_{01}+c_{11}+\frac{1}{2} c_{21}=\frac{1}{18}<1, \\
& r_{2}=3\left[\frac{1}{2}\left(\sum_{i=0}^{2} b_{i}+\sum_{i=1}^{2} d_{i}\right)+c_{11}+c_{21}\right]=\frac{11}{108}<1, \\
& r_{3}=18\left[\frac{3}{4}\left(\sum_{i=0}^{2} b_{i}+\sum_{i=1}^{2} d_{i}\right)+c_{11}+\frac{3}{2} c_{21}\right]=\frac{5}{6}<1 .
\end{aligned}
$$

Thus, (4.2) satisfies all conditions of Theorem 2. It follows that (4.2) has a unique solution.

\section{Competing interests}

The authors declare that they have no competing interests.

\section{Authors' contributions}

All authors contributed equally to the manuscript and typed, read and approved the final manuscript.

\section{Acknowledgements}

The authors are very grateful to the referees for the very useful comments and for detailed remarks that improved the presentation and the contents of the manuscript. This research is supported by the NNSF of China (No. 11171085), the SRF of Hunan Provincial Science and Technology Department (No. 2013FJ3096), the SRF of Hunan Provincial

(No. 13JJ3106), and the SRF of Hunan Provincial Education Department (No. 12C0101).

\section{Received: 1 August 2013 Accepted: 9 January 2014 Published: 27 Jan 2014}

\section{References}

1. Lakshmikantham, V, Bainov, DD, Simeonov, PS: Theory of Impulsive Differential Equations. World Scientific, Singapore (1989)

2. Bainov, DD, Simeonov, PS: Impulsive Differential Equations: Periodic Solutions and Applications. Longman, Harlow (1993)

3. Samoilenko, AM, Perestyuk, NA: Impulsive Differential Equations. World Scientific, Singapore (1995)

4. He, ZM, Yu, JS: Periodic boundary value problem for first-order impulsive functional differential equations. J. Comput. Appl. Math. 138, 205-217 (2002)

5. Luo, ZG, Shen, JH: Stability results for impulsive functional differential equations with infinite delays. J. Comput. Appl. Math. 131, 55-64 (2001)

6. Sun, JT, Zhang, YP: Impulsive control of a nuclear spin generator. J. Comput. Appl. Math. 157, 235-242 (2003)

7. Gurtin, ME, Pipkin, AC: A general theory of heat conduction with finite wave speed. Arch. Ration. Mech. Anal. 31, $113-126(1968)$

8. Nunziato, JW: On heat conduction in materials with memory. Q. Appl. Math. 29, 187-204 (1971)

9. Adimy, M, Ezzimbi, K: A class of linear partial neutral functional-differential equations with nondense domain. J. Differ. Equ. 147, 285-332 (1998)

10. Dako, R: Linear autonomous neutral differential equations in Banach space. J. Differ. Equ. 25, 258-274 (1997)

11. $\mathrm{Fu}, \mathrm{XL}$, Ezzimbi, $\mathrm{K}$ : Existence of solutions for neutral functional differential evolution equations with nonlocal conditions. Nonlinear Anal., Theory Methods Appl. 54, 215-227 (2003)

12. Hernández, E, Henríquez, HR: Existence of periodic solutions of partial neutral functional differential equations with unbounded delay. J. Comput. Appl. Math. 221, 499-522 (1998)

13. Hernández, E, Henríquez, HR: Existence results for partial neutral functional differential equations with unbounded delay. J. Comput. Appl. Math. 221, 452-475 (1998)

14. Hernández, E, Mckibben, MA: Some comments on: existence of solutions of abstract nonlinear second-order neutral functional integrodifferential equations. Comput. Math. Appl. 50, 655-669 (2005)

15. Agarwal, R, Diagana, T, Hernández, E: Weighted pseudo almost periodic solutions to some partial neutral functional differential equations. J. Nonlinear Convex Anal. 8, 397-415 (2007)

16. Baghli, S, Benchohra, M: Perturbed functional and neutral functional evolution equations with infinite delay in Fréchet spaces. Electron. J. Differ. Equ. 2008,69 (2008)

17. Hernández, E, Rabelo, M, Henríquez, HR: Existence of solutions for impulsive partial neutral functional differential equations. J. Comput. Appl. Math. 331, 1135-1158 (2007)

18. Hernández, E, Mckibben, MA, Henríquez, HR: Existence results for abstract impulsive second order neutral functional differential equations. Nonlinear Anal., Theory Methods Appl. (2008). doi:10.1016/j.na.2008.03.062

19. Benchohra, M, Ouahab, A: Impulsive neutral functional differential equations with variable times. Nonlinear Anal., Theory Methods Appl. 55, 679-693 (2003)

20. Hernández, E, Henríquez, HR: Impulsive partial neutral differential equations. Appl. Math. Lett. 19, $215-222$ (2006) 
21. Chang, YK, Anguraj, A, Arjunan, MM: Existence results for impulsive neutral functional differential equations with infinite delay. Nonlinear Anal. Hybrid Syst. 2, 209-218 (2008)

22. Li, M, Han, M: Existence for neutral impulsive functional differential equations with nonlocal conditions. Indag. Math. 20, 435-451 (2009)

23. Balachandran, K, Annapoorani, N: Existence results for impulsive neutral evolution integrodifferential equations with infinite delay. Nonlinear Anal. Hybrid Syst. 3, 674-684 (2009)

24. Cuevas, C, Hernández, E, Rabelo, M: The existence of solutions for impulsive neutral functional differential equations. Comput. Math. Appl. 58, 744-757 (2009)

25. Anguraj, A, Karthikeyan, K: Existence of solutions for impulsive neutral functional differential equations with nonlocal conditions. Nonlinear Anal., Theory Methods Appl. 70, 2717-2721 (2009)

26. Park, JY, Balachandran, $\mathrm{K}$, Annapoorani, N: Existence results for impulsive neutral functional integrodifferential equations with infinite delay. Nonlinear Anal., Theory Methods Appl. 71, 3152-3162 (2009)

27. Chang, YK, Nieto, JJ, Zhao, ZH: Existence results for a nondensely-defined impulsive neutral differential equation with state-dependent delay. Nonlinear Anal. Hybrid Syst. 4, 593-599 (2010)

28. Chang, YK, Kavitha, V, Arjunan, MM: Existence results for impulsive neutral differential and integrodifferential equations with nonlocal conditions via fractional operators. Nonlinear Anal. Hybrid Syst. 4, $32-43$ (2010)

29. Ye, RP: Existence of solutions for impulsive partial neutral functional differential equation with infinite delay. Nonlinear Anal., Theory Methods Appl. 73, 155-162 (2010)

30. Henrández, E, Henríquez, HR, Mckibben, MA: Existence results for abstract impulsive second-order neutral functional differential equations. Nonlinear Anal., Theory Methods Appl. 70, 2736-2751 (2009)

31. Guo, DJ: Nonlinear Functional Analysis. Shangdong Science and Technology Press, Jinan (2002)

32. Fu, XL, Yan, BQ, Liu, YS: Theory of Impulsive Differential System. Science Press, Beijing (2005)

10.1186/1687-1847-2014-38

Cite this article as: $Y$ Ye et al.: Existence results for third-order impulsive neutral differential equations with deviating

arguments. Advances in Difference Equations 2014, 2014:38

\section{Submit your manuscript to a SpringerOpen ${ }^{\ominus}$ journal and benefit from:}

- Convenient online submission

Rigorous peer review

- Immediate publication on acceptance

- Open access: articles freely available online

- High visibility within the field

- Retaining the copyright to your article 